\title{
Transcranial Doppler: An Introduction for Primary Care Physicians
}

\author{
Mounzer Y. Kassab, MD, MA, Arshad Majid, MD, Muhammad U. Farooq, MD, \\ Hend Azhary, MD, Linda A. Hershey, MD, Edward M. Bednarczyk, PharmD, \\ Dion F. Graybeal, MD, and Mark D. Johnson, MD
}

Transcranial Doppler (TCD) is a diagnostic tool that can be used at bedside to assess the cerebral vasculature noninvasively. It is inexpensive, safe, and reliable when compared with other techniques. It can be repeated multiple times and can be used for continuous monitoring if needed. Screening of children with sickle cell disease to assess and prevent ischemic strokes and monitoring for vasospasm after subarachnoid hemorrhage are well established, evidenced based utilizations of TCD. It is useful for the evaluation of occlusive intracranial vascular lesions with many emerging indications in the management of ischemic stroke. TCD with micro-bubble enhancement has comparable sensitivity to transesophageal echocardiogram in detecting right-to-left atrial cardiac shunts. TCD is underused as a clinical tool despite well established indications. The pressure to contain increasing medical cost will likely result in increased utilization of this test in future. ( $\mathrm{J}$ Am Board Fam Med 2007;20:65-71.)

Christian Johann Doppler, a mathematician, physician, and astronomer, first described the Doppler principle in 1843. Its use in neurology was first reported in 1982, ${ }^{1}$ and since then, its convenience and growing range of uses have made it an attractive tool to evaluate the cerebrovascular tree in patients with neurovascular disorders. Transcranial Doppler (TCD) is a noninvasive ultrasound device that allows real-time evaluation of the intracranial cerebral circulation. It is currently under-utilized in part due to the lack of awareness about the diagnostic usefulness of this test. In this article, we will review TCD testing as a procedure and discuss its clinical applications of special interest to the primary care physician.

\footnotetext{
This article was externally peer reviewed.

Submitted 4 August 2006; revised 17 October 2006; accepted 21 October 2006.

From the Department of Neurology and Ophthalmology (MYK, AM, MUF), Michigan State University, East Lansing, MI; Department of Family Practice (HA), Department of Neurology (LAH), Departments of Pharmacy Practice and Nuclear Medicine (EMB), State University of New York at Buffalo, NY; and Department of Neurology (DFG, MD), University of Texas, Southwestern Medical Center, Dallas, TX.

Conflict of interest: none declared.

Corresponding author: Mounzer Y. Kassab, MD, 318 Service Road, A-217 Clinical Center, Michigan State University, East Lansing, MI 48824 (E-mail: mounzer.kassab@ht.msu.edu).
}

\section{Examination and Doppler Instrument}

Ultrasound examination of a vessel by means of TCD is referred to as insonation. The TCD probe is placed over different "acoustic windows" that are specific areas of skull where there is a lack of boney covering or the cranial bone is thin (Figure 1). The transtemporal (temporal) window is used to insonate the middle cerebral artery (MCA), the anterior cerebral artery (ACA), the posterior cerebral artery (PCA), and the terminal portion of the internal carotid artery (TICA), before its bifurcation. The transorbital (orbital) window gives access to insonate the ophthalmic artery (OA) as well as the internal carotid artery at the siphon level. The transforaminal (occipital) window allows insonation of the distal vertebral arteries (VA) and the basilar artery (BA). Finally, the submandibular window allows insonation of the more distal portions of the extracranial internal carotid artery.

TCD does not allow direct visualization of insonated vessels like carotid Doplex, rather it is an indirect evaluation by means of an ultrasonic beam of $2 \mathrm{MHz}$ frequency that is produced from piezoelectric crystals that have been stimulated electrically. This beam bounces off the erythrocytes within the insonated artery. The reflected signal is received by the transducer and converted to an electric signal (Figure 2). This information is subtracted from the transmitted signal and then processed to obtain a waveform that allows accurate 


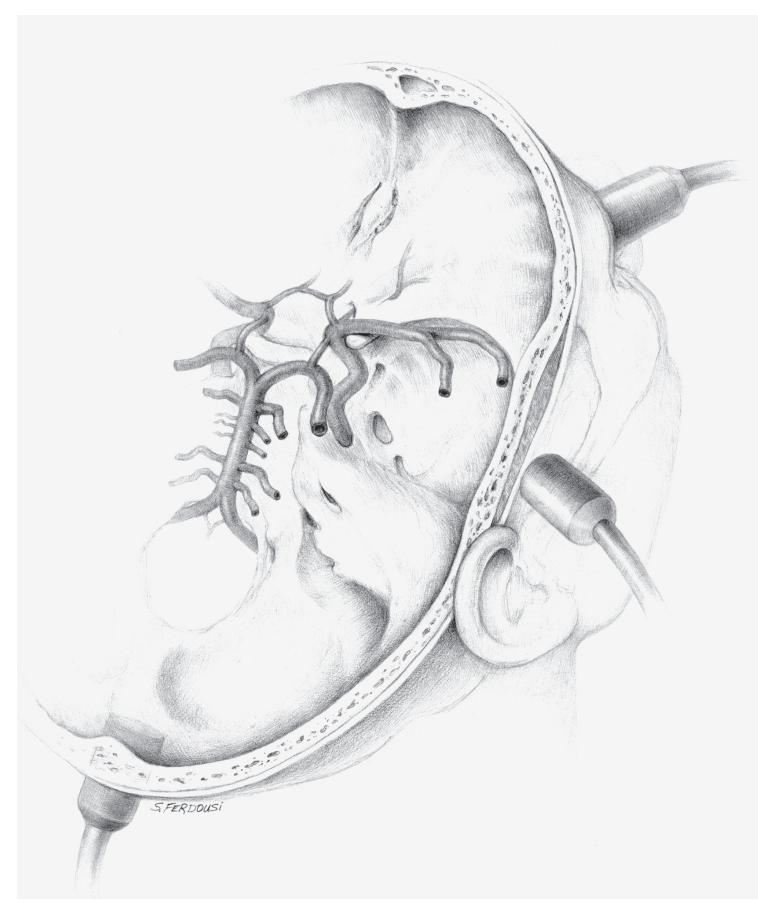

Figure 1. TCD probe positions over different acoustic windows of the skull.

determination of blood flow velocities, direction of flow, and also allows for certain calculated parameters to be added to the evaluation. The pulsatility index (PI) is one of the useful calculated parameters, and it is considered a reliable marker of resistance distal to the insonated site. It is usually calculated by the Gosling equation. ${ }^{2} \mathrm{PI}=($ Peak systolic velocity - end diastolic velocity)/mean velocity.

\section{Introduction to Basic Cerebrovascular Hemodynamics}

The relationship between resistance, pressure, and flow can be expressed by Ohm's law: flow = pres-

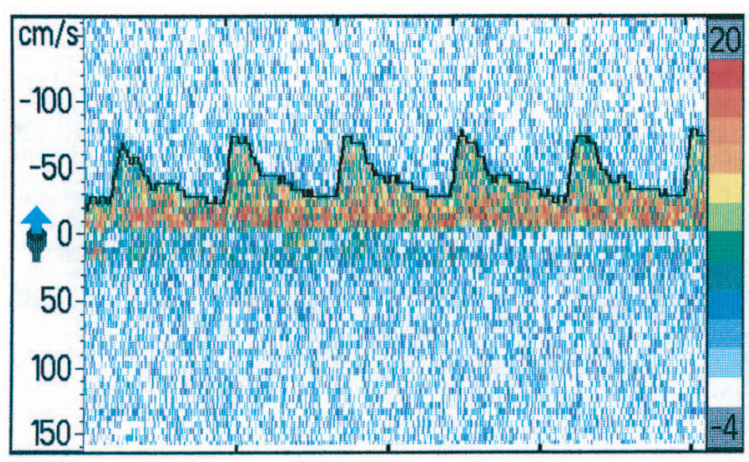

Figure 2. TCD screen waveform with velocity scale on the left. sure/resistance. If we want to apply this relationship to cerebrovascular hemodynamics it translates to cerebral blood flow $=$ cerebral perfusion pressure/cerebrovascular resistance $(\mathrm{CBF}=\mathrm{CPP} /$ CVR).

CPP can be calculated from the mean arterial blood pressure and the intracranial pressure $(\mathrm{CPP}=\mathrm{MAPB}-\mathrm{ICP}) . \mathrm{CVR}$ is influenced in physiologic states by constriction and dilatation of the small size vessels (arterioles) in the brain. In pathologic states, focal changes in resistance can be seen immediately behind an area of significant stenosis.

The main goal of brain cerebrovascular hemodynamics is to keep CBF stable despite alterations in CPP and CVR. This is usually achieved by vascular autoregulation, a process that lowers resistance by inducing vasodilatation to maintain CBF when CPP is decreased. In cases of increased CPP (like increased BP), increasing resistance by vasoconstriction occurs, and thus CBF is maintained.

\section{TCD Examination and Interpretation of Findings}

Different depth range, flow direction, and normal age-related flow velocity (FV) ranges have been established for each vessel. The vessels are identified using these parameters for each of the windows already discussed. Table 1 is a simplified illustration of these values. ${ }^{3}$ TCD measurements are influenced by different physiologic and pathologic factors and by vasoactive medications (Table 2).

\section{Basic Observations Regarding Blood FVs and PI in Different Vascular Scenarios}

1. Pure focal narrowing at the site of insonation will cause an increase in FV.

2. Narrowing or obstructing lesions proximal to the insonation site will cause a decrease in FV observed at the insonation site.

3. Downstream (distal) decrease in vascular resistance (as in the presence of arteriovenous malformation will increase FV and decrease PI at the site of insonation.

4. Downstream increased vascular resistance (as in stenosis or obstruction) will decrease FV and increase PI proximal to lesion.

\section{Clinical Applications}

The clinical applications of TCD are growing. The American Academy of Neurology published TCD guidelines for clinicians. ${ }^{4}$ Although directed to 


\begin{tabular}{|c|c|c|c|c|}
\hline Artery & Window & Depth (mm) & Direction & $\begin{array}{c}\text { Mean Flow } \\
\text { Velocity }\end{array}$ \\
\hline MCA & Temporal & 30 to 60 & Toward probe & $55 \pm 12 \mathrm{~cm} / \mathrm{s}$ \\
\hline ACA & Temporal & 60 to 85 & Away & $50 \pm 11 \mathrm{~cm} / \mathrm{s}$ \\
\hline PCA & Temporal & 60 to 70 & Bidirectional & $40 \pm 10 \mathrm{~cm} / \mathrm{s}$ \\
\hline TICA & Temporal & 55 to 65 & Toward & $39 \pm 09 \mathrm{~cm} / \mathrm{s}$ \\
\hline ICA (siphon) & Orbital & 60 to 80 & Bidirectional & $45 \pm 15 \mathrm{~cm} / \mathrm{s}$ \\
\hline $\mathrm{OA}$ & Orbital & 40 to 60 & Toward & $20 \pm 10 \mathrm{~cm} / \mathrm{s}$ \\
\hline VA & Occipital & 60 to 80 & Away & $38 \pm 10 \mathrm{~cm} / \mathrm{s}$ \\
\hline BA & Occipital & 80 to 110 & Away & $41 \pm 10 \mathrm{~cm} / \mathrm{s}$ \\
\hline
\end{tabular}

TCD, transcranial Doppler; MCA, middle cerebral artery; ACA, anterior cerebral artery; PCA, posterior cerebral artery; TICA, terminal internal carotid artery; ICA, internal carotid artery; OA, ophthalmic artery; VR, vertebral artery; BA, basilar artery.

neurologists, these guidelines may be useful for the primary care physician.

\section{Sickle Cell Disease}

This may be one of the most important TCD applications, and it is of very special interest for the primary care physician. Recent guidelines regard the use of $\mathrm{TCD}^{4}$ as a type A level of evidence (established as useful predictive for suspected condition), and class I (evidence provided by prospective studies in broad spectrum of persons with suspected condition) screening tool to assess stroke risk in children aged 2 to 16 years with sickle cell disease.

Sickle cell disease is associated with progressive occlusion of large intracranial arteries (most frequently the intracranial ICA and MCA). Total occlusion of any one of these arteries will lead to a massive stroke. These arteries are very accessible to TCD insonation and TCD can monitor FVs over time.
According to the TCD criteria for sickle cell disease, a MFV of up to $170 \mathrm{~cm} / \mathrm{s}$ is considered normal and a MFV of 171 to $199 \mathrm{~cm} / \mathrm{s}$ is called "conditional." MFV of equal or greater than 200 $\mathrm{cm} / \mathrm{s}$ is considered abnormal and require transfusion. ${ }^{5}$

A mean FV (MFV) of $200 \mathrm{~cm} / \mathrm{s}$ or greater is accompanied by a stroke risk of $40 \%$ within the next 3 years. Transfusion, with reduction of hemoglobin $\mathrm{S}$ to less than $30 \%$ of total hemoglobin, will lower this risk by $70 \%$ compared with standard care alone..$^{5-8}$ An optimal timing to re-screen children with sickle cell disease and normal TCD is not set, but a repeat TCD examination every 6 months seems to be a reasonable objective.

\section{Intracranial Vasospasm}

Intracranial vasospasm is the constriction of cerebral blood vessels due to the presence of blood in the subarachnoid space after a trauma or rupture of cerebral aneurysm. The vasospasm-if severe-

Table 2. Effects of Different Physiological States on TCD Flow Velocity

\begin{tabular}{lc}
\hline Physiologic Factor & Effects \\
\hline Increase age & Decrease FV \\
Increase CSF pressure & Decrease FV \\
Increase central venous pressure & Decrease FV \\
Increase $\mathrm{PaCO}_{2}$ & Increase FV due to vasodilatation \\
Increase cardiac output & Decrease FV to maintain normal CBF \\
Increased blood viscosity & Decrease FV \\
Anemia & Increase FV \\
Drugs with vasodilatation properties & Increase FV \\
Drugs with vasoconstriction properties & Decrease FV \\
\hline
\end{tabular}

TCD, transcranial Doppler; CSF, cerebrospinal fluid; FV, flow velocity; CBF, cerebral blood flow. 
may result in ischemia to the brain and is associated with an increase in mortality of 1.5 - to 3 -fold during the first 2 weeks after subarachnoid hemorrhage (SAH). ${ }^{9}$ Vasospasm typically occurs within 3 to 21 days after SAH and may last for 12 to 16 days. ${ }^{10}$ Clinically this manifests a deterioration of the patient's level of consciousness, new focal neurological signs, and headaches.

The pathophysiological changes that underlie vascular constriction after SAH is not completely known but may include changes within the vessel walls themselves, alteration of levels of vasoactive substances, and broader pathologic conditions such as immune responses, inflammation, and oxidative damage.

Subarachnoid hemorrhage occurs in an estimated 25,000 to 30,000 people in the United States each year. ${ }^{10}$ It could be identified by TCD up to 1 to 2 days before it becomes clinically symptomatic allowing for immediate initiation of triple $\mathrm{H}$ therapy.

Vasospasm that follows SAH causes increased FV inside intracranial vessels. This can be detected by TCD indicating the need for treatments before the onset of ischemia. TCD is already in use to diagnose and monitor management of vasospasm in emergency and critical settings according to established guidelines. ${ }^{11-16}$ Usually a baseline TCD is performed at the earliest possible time (day 0); a daily TCD is then performed during days 3 to 10 . A mean FV of $120 \mathrm{~cm} / \mathrm{s}$ is considered a sign of mild spasm and a value above $180 \mathrm{~cm} / \mathrm{s}$ is considered a sign of severe spasm. Mean FV obtained by TCD was found to correlate well with the angiographic residual lumen diameter of the MCA, and it was found to have excellent specificity (100\%) but only good sensitivity $(58.6 \%)$ in diagnosing vasospasm only due to the involvement of vessels that are not evaluated by TCD at times. ${ }^{13}$ The accuracy of TCD in the diagnosis of cerebral vasospasm can be increased by using the Lindegaard ratio (see Table 3). By calculating the MFV ratio between MCA, $\mathrm{ACA}$, and the ipsilateral ICA $\left(\mathrm{V}_{\mathrm{MCA}} / \mathrm{V}_{\mathrm{ICA}}\right.$ or $\mathrm{V}_{\mathrm{ACA}} /$ $\mathrm{V}_{\text {ICA }}$ ), changes due to generalized hyperemia can be distinguished from vasospasm. ${ }^{17}$

\section{Arterial Stenosis and Occlusion}

TCD measurements that correlate with stenosis would be increase $\mathrm{FV}$ at the stenotic site.

1. Decrease FV downstream from a stenotic site.
Table 3. TCD's Mean Flow Velocity Criteria for Cerebral Vasospasm

\begin{tabular}{lcc}
\hline $\begin{array}{l}\text { Severity of } \\
\text { Vasospasm }\end{array}$ & $\begin{array}{c}\text { MFV Value } \\
\mathrm{cm} / \mathrm{s}\end{array}$ & $\begin{array}{c}\text { MCA/ICA } \\
\text { Ratio }\end{array}$ \\
\hline Normal & $<85$ & $<3$ \\
Mild & $<120$ & $<3$ \\
Moderate & 120 to 150 & 3 to 5.9 \\
Severe & 151 to 200 & $>6$ \\
Critical & $>200$ & $>6$ \\
\hline
\end{tabular}

TCD, transcranial Doppler; MFV, mean flow velocity; MCA, middle cerebral artery; ICA, internal carotid artery.

2. Decrease FV proximal and increase PI proximal to the stenotic site.

3. Increase FV and/or reverse flow in collateral vessels (like the finding of reverse blood flow in the $\mathrm{OA}$ in case of ipsilateral carotid artery occlusion and the finding of increased FV of the first segment of contralateral ACA shunting blood through the anterior communicating artery to the contralateral hemisphere in the same scenario).

In a case of total occlusion, there should be no flow signal from the occluded site. Increased velocity and/or reversed flow in the collateral vessels may also be seen. Other vessels in the same window should be accessible for insonation. A decrease in blood FV proximal to the site of the occlusion may also be seen. The presence of multiple findings described above increase the likelihood of the presence of stenosis or occlusion in a given vessel. ${ }^{18}$

Similar TCD approaches are already utilized in some centers for the detection of occluded vessels in acute and subacute stroke. It is the noninvasive test of choice to evaluate the cerebrovascular tree when a contraindication exists to the use of MRI and CT and cerebral angiography. TCD testing has a high positive predictive value of $>80 \%{ }^{19}$ making TCD an attractive tool for assessing the cerebrovascular tree in low risk patients.

In the Midwestern region of the United States, Medicare pays $\$ 62.31$ for the professional component and $\$ 465.30$ for the technical component of MRA of the intracranial vessels and pays additional and a similar amount for MRA of the cervical vessels. It only pays $\$ 51.99$ for the professional component and $\$ 245.47$ for a complete TCD study (for both intracranial and extracranial vessels). Since TCD is a noninvasive $e^{20,21}$ and inexpensive technique with no known risks or side effects, it can be 
an excellent tool for frequent follow-up of vascular lesions to assess effectiveness of a therapy without the concern of subjecting the patient to frequent radiation and the increasing costs of diagnostic testing like MRI.

TCD can also be used to observe the effectiveness of thrombolytic treatment in stroke patients. If the vessel is not recannulized, other treatments can be considered. $^{21}$

\section{Monitoring for Sources of Emboli and Heart Shunts during Procedures}

Microemboli traveling along an insonated vessel will appear as high intensity transient signals (HITS) on the TCD spectrum. Although the exact clinical relevance of these findings remains uncertain, it may identify high-risk status for clinical stroke offering an opportunity to localize the source in patients with multiple potential sources and providing a possible monitoring tool for the efficacy of a chosen treatment.

TCD can also be used as a quality control, training, and monitoring tool inside operation rooms for surgeries with risk of embolization ${ }^{22-24}$ their contribution to the postoperative neurobehavioral changes.

TCD can detect the presence of right-to-left shunts such as patent foramen ovale (PFO) in patients with contraindications to transesophageal echo (TEE) with similar sensitivity and specifici$\mathrm{ty}^{25-28}$. This clinic use requires the administration of agitated saline after which the patient performs a valsalva maneuver ${ }^{28}$. The test is positive for rightto-left shunt if a shower of high signal material (air) is detected in the MCA by TCD 5 to 10 seconds after the intravenous injection of $10 \mathrm{~mL}$ of agitated saline $^{26}$. If this shower of air emboli is detected after a minute of injection, it might indicate the presence of a pulmonary shunt; a diagnosis that cannot be obtained by TEE.

\section{Brain Death (cerebral circulatory arrest)}

TCD evaluation of a brain death patient yields a "reverberating or oscillating" pattern of flow (normal arterial blood flow in systole and reversed flow in diastole secondary to the very high distal brain resistant in the brain dead patients) leading to absence of net flow per unit of time. ${ }^{29-32}$ The reverberating pattern may progress to "no flow" in the advanced stages of brain death. Although it is not a common practice to get a TCD as part of a brain death examination, it can, however, be used as a useful adjunct test. ${ }^{33}$

\section{Testing for Cerebrovascular Autoregulation}

Cerebral autoregulation is the ability to maintain the cerebral blood flow despite minute-to-minute variation in cerebral perfusion rate. In a normal physiologic state, this can be achieved by the arteriolar control over the cerebral peripheral vascular resistance. People with impaired cerebral autoregulation might have a variety of presentations such as headache, dizziness, and syncope.

TCD measurements of FV and PI before and during pharmacological or mechanical manipulation of autoregulation and systemic blood pressure can be used to monitor the reactivity of the intracranial vasculature tree. Breath-holding and acetazolamide administration are two of the most used maneuvers to manipulate cerebral autoregulation. $^{34-36}$

The breath-holding test is used to assess cerebrovascular reactivity to hypercapnia as calculated by means of the breath-holding index (BHI). The index is calculated by dividing the percent increase in mean FV (MFV) during breath-holding by the breath-holding time (in seconds) that subjects held their breath after a normal inspiration: BHI = $\{[M F V$ at the end of breath-holding - resting MFV]/resting MFV $\times(100 /$ seconds of breathholding).

This technique can be useful in testing the candidacy of asymptomatic patients with internal carotid artery stenosis for endarterectomy surgery. Recent data ${ }^{37}$ showed increased risk of ischemic strokes in patients with severe $(>70 \%)$ carotid stenosis and impaired autoregulation as tested by TCD. TCD can also assess the potential risk of cerebral ischemia in patients with carotid stenosis going for major anesthesia and surgery. TCD might also be a useful investigation in work up of a patient with vasovagal syncope $\mathrm{s}^{38-41}$.

\section{Conclusion: Advantages and Limitations of TCD}

TCD is relatively inexpensive, noninvasive, portable and fairly easy to use. It allows frequent repeated measurements and continuous monitoring. Immediate, real time detection of changes in cerebrovascular hemodynamics is possible. It can be utilized by any medical specialty to evaluate several neurovascular disorders. 
In many communities (especially rural ones), there is no neurologist and/or MRI machine available for appropriate stroke workup. In those settings and in many other places a "complete" stroke workup consists of carotid Doppler and two-dimensional echocardiogram. This misses the important evaluation of intracranial vasculature. A TCD may be the inexpensive, simple mean to determine which patients must be referred to a specialized center for further evaluation. In addition, and in patients who decline intervention initially, TCD gives a tool for monitoring the identified lesions through the years and evaluate the effectiveness of medical treatment.

Finally, TCD may be the only mean possible to evaluate intracranial vessels in cases when other radiographic means are contraindicated. TCD is a "blind procedure"; its accuracy relies on the knowledge and experience of a trained technician and interpreter. It has limited ability to detect distal branches of intracranial vessels. In $5 \%$ to $10 \%$ of cases, sufficient penetration of the bone window can not be achieved for ample insonation.

A certificate in neurosonology (TCD and carotid Doppler) is granted by the American neuroimaging society after specific training requirements are met and an examination is passed.

\section{References}

1. Aaslid R, Markwalder TM, Nornes H. Noninvasive transcranial Doppler ultrasound recording of flow velocity in basal cerebral arteries. J Neurosurg 1982; 57:769-74.

2. Padayachee TS, Kirkham FJ, Lewis RR, Gillard J, Hutchinson MC, Gosling RG. Transcranial measurement of blood velocities in the basal cerebral arteries using pulsed Doppler ultrasound: a method of assessing the Circle of Willis. Ultrasound Med Biol 1986;12:5-14.

3. Ringelstein EB, Kahlscheuer B, Niggemeyer E, Otis SM. Transcranial Doppler sonography: anatomical landmarks and normal velocity values. Ultrasound Med Biol 1990;16:745-61.

4. Sloan MA, Alexandrov AV, Tegeler CH, et al. Assessment: transcranial Doppler ultrasonography: report of the Therapeutics and Technology Assessment Subcommittee of the American Academy of Neurology. Neurology 2004;62:1468-81.

5. Adams RJ, McKie VC, Hsu L, et al. Prevention of a first stroke by transfusions in children with sickle cell anemia and abnormal results on transcranial Doppler ultrasonography. N Engl J Med 1998;339:5-11.

6. Adams RJ, McKie VC, Carl EM, et al. Long-term stroke risk in children with sickle cell disease screened with transcranial Doppler. Ann Neurol 1997;42:699-704.

7. Adams RJ, McKie VC, Brambilla D, et al. Stroke prevention trial in sickle cell anemia. Control Clin Trials 1998;19:110-29.

8. Adams R, McKie V, Nichols F, et al. The use of transcranial ultrasonography to predict stroke in sickle cell disease. N Engl J Med 1992;326:605-10.

9. Treggiari-Venzi MM, Suter PM, Romand JA. Review of medical prevention of vasospasm after aneurysmal subarachnoid hemorrhage: a problem of neurointensive care. Neurosurgery 2001;48:249-61; Discussion 261-2.

10. Oyama K, Criddle L. Vasospasm after aneurysmal subarachnoid hemorrhage. Crit Care Nurse 2004;24: 58-60, 62, 64-7.

11. Aaslid R, Huber P, Nornes H. Evaluation of cerebrovascular spasm with transcranial Doppler ultrasound. J Neurosurg 1984;60:37-41.

12. Grosset DG, Straiton J, du Trevou M, Bullock R. Prediction of symptomatic vasospasm after subarachnoid hemorrhage by rapidly increasing transcranial Doppler velocity and cerebral blood flow changes. Stroke 1992;23:674-9.

13. Sloan MA, Haley EC, Jr., Kassell NF, et al. Sensitivity and specificity of transcranial Doppler ultrasonography in the diagnosis of vasospasm following subarachnoid hemorrhage. Neurology 1989;39: $1514-8$.

14. Harders AG, Gilsbach JM. Time course of blood velocity changes related to vasospasm in the circle of Willis measured by transcranial Doppler ultrasound. J Neurosurg 1987;66:718-28.

15. Aaslid R, Huber P, Nornes H. A transcranial Doppler method in the evaluation of cerebrovascular spasm. Neuroradiology 1986;28:11-6.

16. Grosset DG, Straiton J, McDonald I, Cockburn M, Bullock R. Use of transcranial Doppler sonography to predict development of a delayed ischemic deficit after subarachnoid hemorrhage. J Neurosurg 1993; 78:183-7.

17. Lindegaard KF, Nornes H, Bakke SJ, Sorteberg W, Nakstad P. Cerebral vasospasm after subarachnoid haemorrhage investigated by means of transcranial Doppler ultrasound. Acta Neurochir Suppl (Wien) 1988;42:81-4.

18. Demchuk AM, Christou I, Wein TH, et al. Accuracy and criteria for localizing arterial occlusion with transcranial Doppler. J Neuroimaging 2000;10:1-12.

19. Alexandrov AV, Demchuk AM, Wein TH, Grotta JC. Yield of transcranial Doppler in acute cerebral ischemia. Stroke 1999;30:1604-9.

20. Constantoyannis C, Kourtopoulos H. [Transcranial Doppler in neurosurgery. A non-invasive method in the diagnosis of vasospasm and subarachnoid hemorrhage]. Lakartidningen 2002;99:1686-90.

21. Alexandrov AV, Demchuk AM, Felberg RA, Grotta JC, Krieger DW. Intracranial clot dissolution is as- 
sociated with embolic signals on transcranial Doppler. J Neuroimaging 2000;10:27-32.

22. Albin MS HC, Mitzel H, Bunegin L, Gover F. Aeric microemboli and the transcranial doppler (TCD): episodic frequency and timing in 62 cases of open heart surgery. J Neurosurg Anesthesiol 1991;75:53.

23. Smith JL, Evans DH, Gaunt ME, London NJ, Bell PR, Naylor AR. Experience with transcranial Doppler monitoring reduces the incidence of particulate embolization during carotid endarterectomy. $\mathrm{Br} \mathrm{J}$ Surg 1998;85:56-9.

24. Clark RE, Brillman J, Davis DA, Lovell MR, Price TR, Magovern GJ. Microemboli during coronary artery bypass grafting. Genesis and effect on outcome. J Thorac Cardiovasc Surg 1995;109:249-57; Discussion 257-8.

25. Nemec JJ, Marwick TH, Lorig RJ, et al. Comparison of transcranial Doppler ultrasound and transesophageal contrast echocardiography in the detection of interatrial right-to-left shunts. Am J Cardiol 1991;68:1498-502.

26. Droste DW, Reisener M, Kemeny V, et al. Contrast transcranial Doppler ultrasound in the detection of right-to-left shunts. Reproducibility, comparison of 2 agents, and distribution of microemboli. Stroke 1999;30:1014-8.

27. Klotzsch C, Janssen G, Berlit P. Transesophageal echocardiography and contrast-TCD in the detection of a patent foramen ovale: experiences with 111 patients. Neurology 1994;44:1603-6.

28. Zanette EM, Mancini G, De Castro S, Solaro M, Cartoni D, Chiarotti F. Patent foramen ovale and transcranial Doppler. Comparison of different procedures. Stroke 1996;27:2251-5.

29. Petty GW, Mohr JP, Pedley TA, et al. The role of transcranial Doppler in confirming brain death: sensitivity, specificity, and suggestions for performance and interpretation. Neurology 1990;40:300-3.

30. Feri M, Ralli L, Felici M, Vanni D, Capria V. Transcranial Doppler and brain death diagnosis. Crit Care Med 1994;22:1120-6.

31. Ropper AH, Kehne SM, Wechsler L. Transcranial Doppler in brain death. Neurology 1987;37:1733-5.
32. Hassler W, Steinmetz H, Pirschel J. Transcranial Doppler study of intracranial circulatory arrest. J Neurosurg 1989;71:195-201.

33. Lopez-Navidad A, Caballero F, Domingo P, et al. Early diagnosis of brain death in patients treated with central nervous system depressant drugs. Transplantation 2000;70:131-5.

34. Muller M, Voges M, Piepgras U, Schimrigk K. Assessment of cerebral vasomotor reactivity by transcranial Doppler ultrasound and breath-holding. A comparison with acetazolamide as vasodilatory stimulus. Stroke 1995;26:96-100.

35. Markus HS, Harrison MJ. Estimation of cerebrovascular reactivity using transcranial Doppler, including the use of breath-holding as the vasodilatory stimulus. Stroke 1992;23:668-73.

36. Piepgras A, Schmiedek P, Leinsinger G, Haberl RL, Kirsch CM, Einhaupl KM. A simple test to assess cerebrovascular reserve capacity using transcranial Doppler sonography and acetazolamide. Stroke 1990;21:1306-11.

37. Silvestrini M, Vernieri F, Pasqualetti P, et al. Impaired cerebral vasoreactivity and risk of stroke in patients with asymptomatic carotid artery stenosis. JAMA 2000;283:2122-7.

38. Schondorf R, Benoit J, Wein T. Cerebrovascular and cardiovascular measurements during neurally mediated syncope induced by head-up tilt. Stroke 1997;28:1564-8.

39. Ladwig S, Ries S, Henning O, Valikovics A, Daffertshofer M, Pohlmann-Eden B. Combined electroencephalography and measurements of transcranial blood flow velocity during orthostatic testing-a new approach to assess syncope of unknown origin? Clin Auton Res 1997;7:305-9.

40. Diehl RR, Linden D, Chalkiadaki A, Ringelstein EB, Berlit P. Transcranial Doppler during neurocardiogenic syncope. Clin Auton Res 1996;6:71-4.

41. Fredman CS, Biermann KM, Patel V, Uppstrom EL, Auer AI. Transcranial Doppler ultrasonography during head-upright tilt-table testing. Ann Intern Med 1995;123:848-9. 